\title{
Inspiration, inoculation, and introductions are all critical to successful mentorship for undergraduate women pursuing geoscience careers
}

\author{
Paul R. Hernandez (i) ${ }^{1 凶}$, Amanda S. Adams ${ }^{2}$, Rebecca T. Barnes (i) ${ }^{3}$, Brittany Bloodhart ${ }^{4}$, Melissa Burt (i) ${ }^{5}$, \\ Sandra M. Clinton ${ }^{2}$, Wenyi Du ${ }^{6}$, Heather Henderson ${ }^{6}$, Ilana Pollack ${ }^{5} \&$ Emily V. Fischer ${ }^{5}$
}

Diversity in the geosciences is low despite efforts to improve the representation of different groups in society, for example in terms of gender. Specifically, women are underrepresented in recruitment and retention at every stage of the academic to professional pipeline. Mentoring programs can improve women's motivation and persistence in science, technology, engineering and mathematics (STEM) career pathways. However, mentorship programs consist of multiple components that vary in complexity and cost, which can limit scalability. Here we present results from a randomized experiment with 158 undergraduate women majoring in a geoscience field to identify the critical elements of a successful mentorship program. The combination of three factors was necessary to increase mentoring, motivation, and persistence: inspiration through exposure to geoscience careers via female role models, inoculation through training on how to grow their mentor network and overcome obstacles, and an introduction to a local female geoscientist mentor.

\footnotetext{
${ }^{1}$ Department of Teaching, Learning, \& Culture, College of Education and Human Development, Texas A\&M University, 4232 TAMU, College Station, 778434232 TX, USA. ${ }^{2}$ Department of Geography \& Earth Sciences, UNC Charlotte, McEniry 324, 9201 University City Boulevard, Charlotte, 28223-0001 NC, USA. ${ }^{3}$ Environmental Studies Program, Colorado College, 14 East Cache La Poudre Street, Colorado Springs, 80903-3298 CO, USA. ${ }^{4}$ Department of Psychology, California State University, San Bernardino, 5500 University Parkway, San Bernardino, 92407 CA, USA. ${ }^{5}$ Department of Atmospheric Science, Colorado State University, 200 West Lake Street, 1371 Campus Delivery, Fort Collins, 80523-1371 CO, USA. 6 Department of Learning Sciences and Human

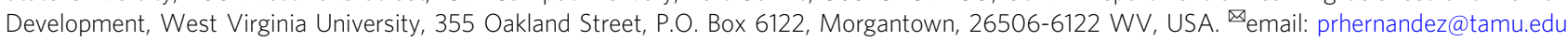


$\mathrm{R}$ ecent research points to a persistent disparity in the representation and inclusion of individuals in the geosciences based on gender, ability status, ethnicity and race, and sexual orientation ${ }^{1-13}$. Mentoring and role modeling are recognized as part of a solution for achieving greater recruitment and retention of students from the underrepresented groups in science, technology, engineering, and mathematics (STEM) fields, such as the geosciences ${ }^{3,14-21}$. In recent years, numerous mentoring and role modeling programs have been implemented to support aspiring women in the geosciences at various early career stages, including at the undergraduate, graduate, and postdoctoral levels ${ }^{16,22-30}$. Furthermore, recent advancements in mentoring theory point to the importance of developing a network with multiple mentoring relationships (relative to none or one relationship) for degree and career success, especially for women in male-dominated fields ${ }^{29-33}$. Social science research indicates that role models and mentors support persistence in STEM because they inspire and motivate mentees, particularly those from the underrepresented groups, by helping the mentees to see themselves as a scientist and belonging in their field of study (e.g., strengthening their scientific identity), inoculating them from negative social stereotypes, and promoting skills to effectively cope with barriers to STEM careers ${ }^{34-38}$.

PROmoting Geoscience Research, Education, and SuccesS (PROGRESS) is a prototypical multi-component mentoring and role modeling program aimed at supporting first- and secondyear college women interested in geoscience careers $24,29,30$. PROGRESS involves participation in a "kick-off" workshop designed to expose undergraduate women to geoscience careers via interactive panels with diverse female geoscience career role models (Inspiration) ${ }^{36,39,40}$, as well as to provide training on how undergraduates may grow their network of career mentors and develop coping skills to overcome barriers to success in geoscience careers (Inoculation) ${ }^{16,41}$. In addition, PROGRESS facilitates post-workshop introductions between the undergraduate women and local female geoscientists (Introduction) ${ }^{42}$. Longitudinal research indicates that, compared to similar women in a propensity score-matched control group, women in PROGRESS identify more female career role models and develop stronger mentorship networks, which in turn results in the development of a stronger science identity and higher intentions to persist in their scientific career pursuits in the short term (6 months post-workshop). Research also indicates higher rates of persistence for women in PROGRESS, such as seven times higher rates of persistence in a geoscience major in the medium term (up to 18 months post-workshop) ${ }^{29,30}$, and ultimately higher longterm rates of persistence in a geoscience major $(94.7 \%$ vs. $76.3 \%$ persistence rate) or any STEM major $(91.7 \%$ vs. $81.4 \%$ persistence rate) through their senior year of college, compared to matched control group participants.

Despite promising evidence of success and clear links to social science theory, important questions about mentorship programs, such as PROGRESS, remain unanswered. First, social science research has not yet distinguished between benefits associated with identifying inspirational career role models from benefits associated with receiving tangible support from career mentors. That is, the existing role modeling social science literature largely examines benefits associated with relatively low cost interventions focused on reading about or listening to several short inspirational biographies of career role models, with no opportunity to form supportive mentoring relationships ${ }^{43-46}$. Similarly, the existing mentoring literature largely examines benefits associated with tangible relational supports provided to mentees, with no opportunity to experimentally isolate the impact of role modeling from that of mentorship support ${ }^{17,47-50}$. Therefore, one goal of this study was to identify the unique contributions that role models vs. mentors play in college women's persistence in STEM fields.

Second, mentorship programs face replicability challenges due to their multifaceted design. That is, many research-based programs, such as PROGRESS, contain multiple components of varying complexity, resource intensity, and cost, which preclude the identification of essential components for success ${ }^{50}$. The complexity of successful programs can also make transferability to and replicability by new implementers more challenging and ultimately can hamper scalability and sustainability ${ }^{50-52}$. Therefore, the second goal of this study was to isolate and evaluate the most critical components of PROGRESS to address scalability and sustainability.

To meet these goals, we conducted a randomized experiment to identify the combination of elements required to directly promote undergraduate women's role modeling and mentoring experiences, as well as to indirectly promote scientific identity, coping skills, and ultimately persistence intentions, see Fig. 1 (Conceptual model). That is, we randomly assigned participants to one of the three workshops and we systematically varied workshop content and post-workshop experiences from the least to the most resource-intensive (i.e., Inspiration, Inspiration \& Inoculation, Inspiration, Inoculation, \& Introduction) to determine what level of program support is needed to impact positive role modeling and mentoring experiences, as well as downstream persistence-related outcomes. We chose these three components because they are distinct aspects of a recently developed mentoring program ${ }^{24}$, each with a strong foundation in gender or educational psychology. We expose the students to allied female career role models (Inspiration) to increase their sense of belonging ${ }^{39}$. Training on how to overcome obstacles (Inoculation) appears important for academic tenacity ${ }^{53}$, overcoming stereotype threat ${ }^{54}$, and invalidating sexism ${ }^{55}$. Finally, we introduced students to a local female geoscientist mentor (Introduction), as there is some evidence that same-gender mentoring is particularly effective for undergraduate women ${ }^{56,57}$.

We recruited undergraduate women of any rank majoring or intending to major in a geoscience-related field from ten universities (see "Methods" section and Supplementary Data 1). We purposely recruited from a wide range of universities with geoscience undergraduate degree programs, including (a) six large, public, research intensive, primarily White serving institutions (PWI), (b) two large, public, minority serving institutions (i.e., one teaching intensive Hispanic serving institution [HSI] and one research intensive Historically Black College and University), (c) one mid-sized, teaching intensive, PWI, and (d) one small, private, teaching intensive, PWI. All applicants who identified as female or a gender minority (transgender woman or gender non-binary/fluid), who were $\geq 18$ years of age, currently or intending to major in a geoscience-related field, and intending to pursue a geoscience-related career received an email invitation to participate in one of the three 1-day professional development workshops held at local university. A blocked randomization procedure was used to assign applicants within each university to a workshop to ensure that applicants from the same university were distributed equally across conditions. The study team provided transportation to and from the professional development workshops for all participants.

Out of 187 women who participated in the study, we report on a final sample of 158 women who both participated in one of the three possible 1-day $(8 \mathrm{~h})$ professional development workshops and completed pre- and post-workshop surveys. Participants were randomly assigned to one of the three groups: (1) Inspiration: exposure to geoscience careers via female career role models with no post-workshop interactions (2) Inspiration \& Inoculation: which added training on how to both grow their mentorship 


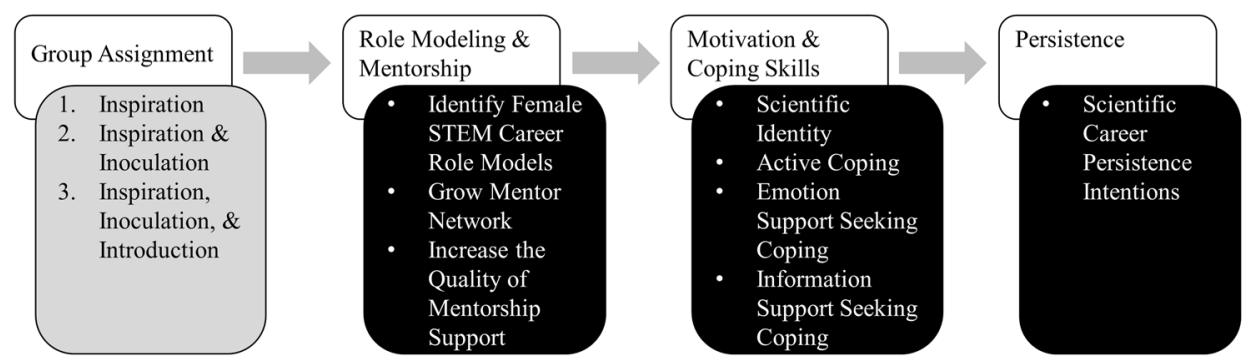

Conceptual Model

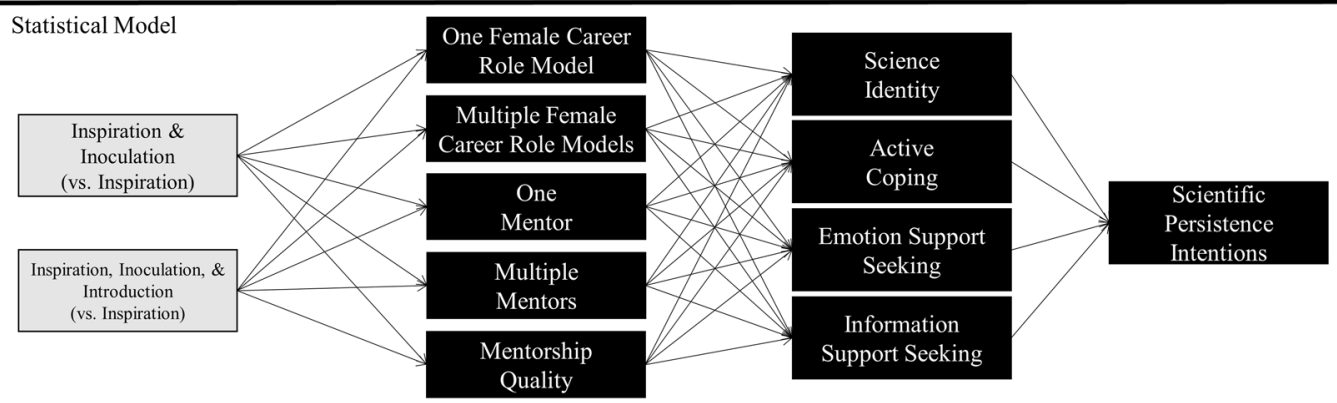

Fig. 1 Experimental process model linking group assignment (gray box) to impacts (black boxes) on persistence through role modeling, mentoring, motivation, and coping skills. Notes: Conceptual model: students were randomly assigned to one of the three experimental groups emphasizing (1) Inspiration (2), Inspiration \& Inoculation, or (3) Inspiration, Inoculation, \& Introductions. We measured and compared the identification of female STEM career role models $(0,1$, or multiple female role models), mentor network size $(0,1$, or multiple mentors), the quality of mentorship support received, science identity (motivation), and coping skills between groups, as well as their persistence intentions. Statistical model: group assignment, posttest role models, and posttest mentors were recoded for analysis, such that each is treated as a binary variable. A number of regression paths are not shown for the sake of parsimony, such that the paths are linking (a) group assignment to motivation \& coping skills and to persistence, (b) role modeling and mentorship to persistence, and (c) pretest role modeling \& mentorship, motivation \& coping skills, and persistence to their posttest counterparts.

network and overcome obstacles to careers in the geosciences with no post-workshop interactions, or (3) Inspiration, Inoculation, \& Introduction: which was identical to the Inspiration \& Inoculation workshop but also included an email from the research team to facilitate a post-workshop introduction to a local female geoscientist (see "Methods" section for complete details). Because prior research suggests that mentoring relationships are more successful when the mentor and mentee perceive a highdegree of shared similarities, a "birds of a feather" procedure was used to match participants with geoscience mentors. The introductory email from the research team highlighted three areas of similar hobbies and interests ${ }^{42}$. All participants completed preand post-workshop surveys focused on the quantity and quality of their career role models and current mentoring relationships (i.e., asked to identify up to three role models and up to three mentors), as well as psychological measures of scientific identity, coping skills, and intentions to pursue a scientific career. Surveys took place 1 week prior to and 3 months after the workshop (see "Methods" section and Supplementary Data 2). A university institutional review board approved all study procedures (Institutional Review Board (IRB\# 14-4829H).

\section{Results}

Workshop differences in role models and mentor networks. Prior to testing our model, we examined the descriptive statistics and particularly the changes in the number of role models and mentors across conditions, Supplementary Data 2. The analysis revealed that women in the Inspiration group showed significant gains in the identification of multiple female STEM career role models from pretest to posttest $(+22.7 \%$, Wilcoxon Signed Rank Test $Z=2.68, p=0.007)$, whereas women in the Inspiration \& Inoculation and the Inspiration, Inoculation, \& Introduction groups did not change from pretest to posttest $(0 \%, Z=0.47, p=$ 0.64 and $-2.1 \%, Z=0.33, p=0.74$, respectively). Furthermore, women in the Inspiration, Inoculation, \& Introduction group showed significant gains having multiple mentors from pretest to posttest $(+20.8 \%, Z=2.64, p=0.008)$, whereas women in the Inspiration and the Inspiration \& Inoculation groups did not change from pretest to posttest $(-7.5 \%, Z=0.17, p=0.86$ and $1.8 \%, Z=0.90, p=0.37$, respectively).

Next, a robust bootstrapped path analysis was used to test the effects of the type of workshop on role modeling, mentoring, and downstream persistence-related outcomes, statistically controlling for pre-workshop levels of these outcomes, see Fig. 1 (Statistical model). All pretest control variables were centered for the analysis. Global model fit statistics indicated that the model provided excellent fit to the data, $\chi_{(\mathrm{df}=86)}^{2}=83.05, p=0.57$, comparative fit index $(\mathrm{CFI})=1.00$, root-mean-square error of approximation $(\mathrm{RMSEA})=0.00$ with $90 \%$ confidence interval (CI) $[0.00,0.04]$, standardized root-mean-square residual $(\mathrm{SRMR})=0.066$. Having achieved excellent model fit, we next evaluated the direct and mediated effects of experimental group assignment on persistence intentions through role modeling, mentoring, and motivation and coping.

The results indicate that women in the "Inspiration" group were more likely to identify multiple female STEM career role models compared to women in the "Inspiration \& Inoculation" and "Inspiration, Inoculation, \& Introduction" groups (Fig. 2a; complete details found in Supplementary Data 3). However, women in the "Inspiration, Inoculation, \& Introduction" group were significantly more likely to report having multiple mentors than those in the "Inspiration" group (Fig. 2b). In addition, women across the three groups did not differ in the quality mentorship support they received from their mentors (Fig. 2c). The mentoring results indicate that variations in the workshop content and post-workshop experiences helped to increase undergraduate women's mentor network but did not necessarily increase the quality of support from mentors within their 


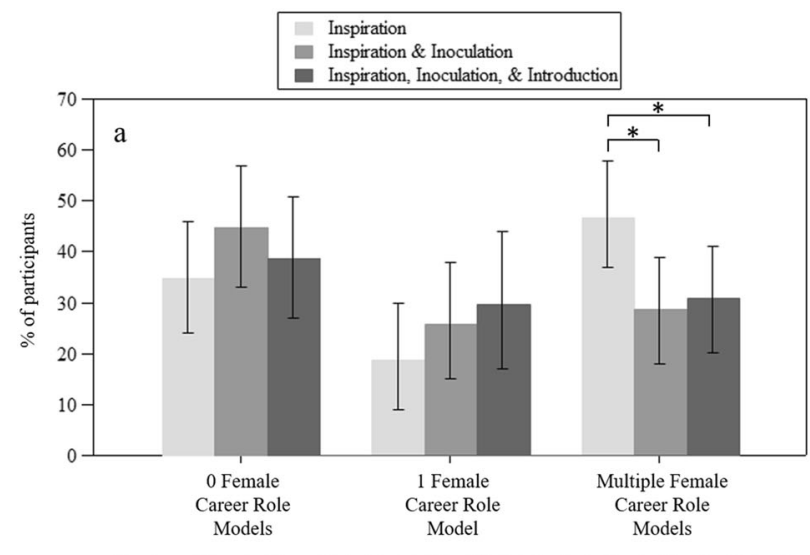

Number of Female Career Role Models Identified at Posttest (Controlling for Pretest)

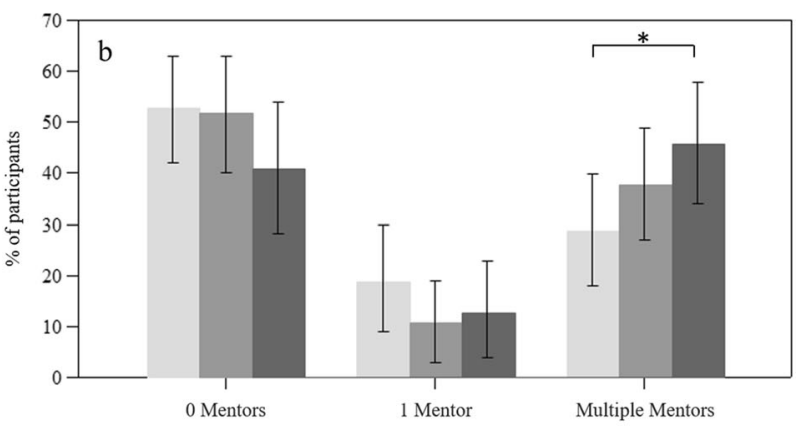

Size of Mentor Network at Posttest (Controlling for Pretest)

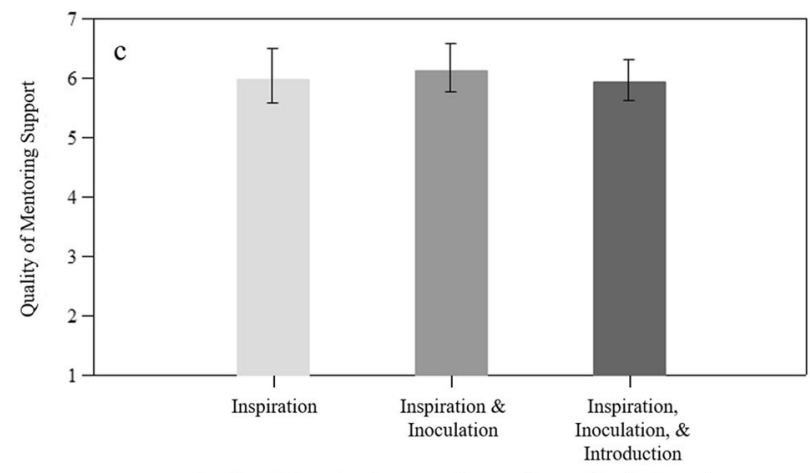

Quality of Mentoring Support at Posttest (Controlling for Pretest)

Fig. 2 Reported mentorship and role models. a The percentage of students reporting 0 , 1, or multiple female career role models, $\mathbf{b}$ percentage of students reporting 0,1 , or multiple mentors, and c average quality mentorship support received as a function of group, controlling for pretest. Notes: Bar graph values represent percentages or means. Error bars reflect the lower and upper limits of bias-corrected bootstrapped 95\% confidence intervals. Asterisks are used to highlight where groups differences are statistically significant.

networks, which was relatively high at both pre- and postworkshop.

Multiple mentors support motivation, resilience, and ultimately persistence. Within the same path analysis model, we next evaluated the degree to which the type of workshop directly and indirectly influenced science identity, coping skills, and persistence intentions through the identification of role models, the size of women's mentor network, or the quality of mentoring support. Consistent with prior research, the model indicated that having multiple mentors exhibited a positive direct effect on posttest science identity, active coping, emotion support seeking coping, and information support seeking coping controlling for pretest (see Fig. 3, complete details in Supplementary Data 3). Contrary to expectations, the model revealed a significant negative direct effect of identifying multiple female role models and a negative direct effect of being in the "Inspiration \& Inoculation" group on posttest science identity, controlling for pretest science identity (see Supplementary Data 3). However, we interpret this as a spurious relationship due to a "classical" statistical suppression effect because identifying multiple role models was uncorrelated with science identity at pretest and posttest but is positively correlated with having multiple mentors (see Supplementary Data 4$)^{58}$. Furthermore, the model also indicated that only posttest scientific identity exhibited a significant positive direct effect on posttest persistence intentions, controlling for pretest intentions (Fig. 3 and Supplementary Data 3).

Finally, the analysis of indirect or mediated effects revealed that women in the "Inspiration, Inoculation, \& Introduction" group developed higher science identity, stronger skills to cope with barriers to success in geoscience careers, and ultimately reported stronger intentions to persist in their scientific career pursuits due to having more support from multiple mentors, compared to women in the "Inspiration" group (i.e., indirect effect tests were statistically significant; see Fig. 3 and Supplementary Data 3).

\section{Discussion}

Although prior research on mentoring programs provides promising evidence for supporting women's pursuit of geoscience degrees and careers ${ }^{3,17,30}$, the existing literature has neither identified which elements or combinations of elements of multicomponent support programs are necessary to reproduce similar results $^{50,51}$ nor clearly distinguished the unique benefits associated with role modeling from those of mentoring. Our randomized experiment indicates that hosting a 1-day professional development workshop emphasizing women's successful journey's into geoscience careers (i.e., Inspiration group) is sufficient to help undergraduate women identify multiple female career role models but is insufficient to help them develop more robust multi-mentor networks. In contrast, facilitating an introduction between an undergraduate and a potential geoscience mentor, in combination with inspiration and inoculation, is necessary to spur growth in the development of multiple supportive mentoring relationships. Although the positive link between social coping and persistence in STEM for college women has been well established $^{59}$, we found that only science identity ultimately predicted greater persistence in STEM in our model. This may be because the large effect of science identity on persistence overwhelmed any effects of coping mechanisms and/or because receiving social support (which is likely a direct consequence of active, emotional, and information-seeking coping skills) is highly related to women's sense of belonging and identity in STEM ${ }^{60}$. We did not hypothesize or test these links, but future research should explore this possibility. Consistent with social science mentoring theory 41 , the development of multi-mentor networks, in turn, promotes higher levels of motivation in the form of scientific identity, coping skills, and intentions to persist in a scientific career. The current findings help to clarify the science of effective mentorship in STEM by showing that the combination of inspiration, inoculation, and introductions are necessary to reproduce findings from prior largely non-experimental research $3,17,30,50$

Despite addressing several challenges related to identifying the essential combination of elements of successful mentoring programs, there are additional factors in need of further study. For example, the present study found that introducing women to a 


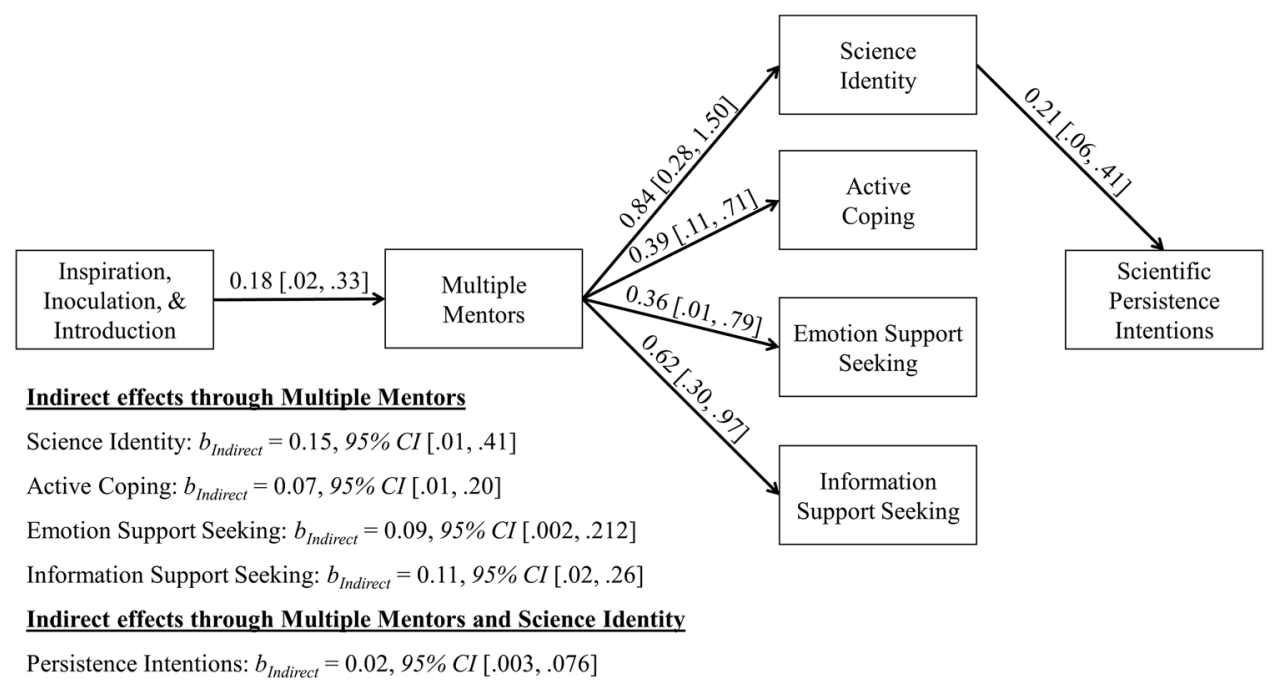

Fig. 3 Indirect effects of being in the "Inspiration, Inoculation, \& Introduction" group on motivation, coping skills, and persistence intentions through multiple mentorships. Notes: Values outside of brackets represent unstandardized regression/path coefficients, while values inside brackets represent the lower and upper limits of bias-corrected bootstrapped 95\% confidence intervals [lower limit, upper limit]. "Inspiration, Inoculation, \& Introduction" is a binary indicator of experimental group status (Inspiration, Inoculation, \& Introduction group $=1$, other type of workshop $=0$ ). "Multiple mentors" is a binary indicator of mentor network size (two or more mentors $=1$, other type of workshop $=0$ ). For the sake of parsimony, only variables and paths associated with statistically significant indirect effects of group status on downstream motivational, coping, and persistence intentions are shown here. Complete details are provided in the "Methods" section.

geoscience mentor was critical to gains in having multiple mentors, which in turn promoted intentions to persist in science. If mentors provide the similar levels of inspiration and inoculation information as is delivered through the workshop, then facilitating introductions with a mentor would provide the most efficient and least resource-intensive way to promote similar results. Future studies should test the relative benefits of Introduction alone vs. Inspiration, Inoculation, \& Introduction. Future work should also quantify the amount of interaction that students had following their introduction to a local same-gender mentor. We kept the mentoring expectation modest for this experiment in part to maximize the commitment from volunteers, but we encouraged productive mentor-mentee pairs to continue interacting. Even with introductions, our rate of mentorship was relatively low (Fig. 2). In all our efforts associated with this project, we have found that many undergraduate women are hesitant to initially engage with mentors, even same-gender mentors within geoscience fields. Furthermore, the present study only paired female undergraduates in geoscience majors with female geoscientists, who are themselves a numerical minority in the profession. Further research is needed to determine the extent to which similar benefits could be achieved by matching female undergraduates with local male geoscientists. In addition, the current study limited the number of potential role models and mentors to a maximum of three persons, which may have censored or put a low ceiling on those variables and influenced our choice of analyses. Future studies should aim to measure role models and mentors in an unrestricted manner in order to treat them as continuous variables and to test whether the cutoffs used in the present study (i.e., none, one, multiple) have equivalent predictive power. Finally, our experiment included undergraduate students from a range of 4-year colleges and universities across two geographic regions. Despite inclusive recruitment efforts and holding half of the workshops at a minority serving institution, most of the undergraduate women who participated in our study were White and cisgender women, thereby preventing us from specifically testing whether this program can benefit women with other intersecting minority identities. Thus the next step is to test whether this approach can ensure that everyone feels they belong in the geosciences. Real progress will also require addressing persistent culture problems in the geosciences that continue to marginalize women and minorities ${ }^{13,61-64}$.

Despite these limitations, this study can inform the development of new mentoring programs, as well as the transferability and sustainability of existing programs. Specifically, our experimental design shows that the benefits associated with a 2-day professional development workshop with follow-up mentoring opportunities can be replicated in a compressed 1-day workshop with follow-up mentoring opportunities ${ }^{30}$. It is also clear that a relatively low-intensity mentoring opportunity infrastructure is sufficient to help undergraduate women grow their mentorship networks through a brief training followed by an email-facilitated introduction to a local similar potential mentor (see "Methods" section for more details). We found a reasonably sized pool of potential mentors by inviting women in a wide range of professional roles and by clearly setting mentoring commitment expectations. Specifically, we recruited women from a wide range of career stages in the geosciences, including post-baccalaureates (graduate, postdoctoral), scientists outside of the academy, and faculty. To reduce demands on volunteer mentors, we asked the pair to meet on one occasion (e.g., $1 \mathrm{~h}$ over coffee), but more interactions were encouraged if the pair felt that a good mentoring relationship could be formed. Finally, pairing students with potential mentors was based on a scalable "birds of a feather" approach, where matching is based on shared interest discovered through a brief "getting to know you" survey. Together, these practices are easily adoptable for STEM organizations looking to increase diversity and could immediately be widely implemented to better support undergraduate women.

\section{Methods}

Participants. In spring 2019, 187 undergraduate college women in geoscience majors from 10 universities from 2 regions of the U.S. participated in a 1-day regional professional development workshop. Undergraduates were invited to participate in the workshop if they met the following inclusion criteria: (a) they were $\geq 18$ years of age, (b) they self-identified as female, (c) they were currently pursuing or planning to pursue a major in a geoscience-related field, and (d) they 
were interested in pursuing an Earth Systems or Environmental Science career. As described in the main text, participants were randomly assigned to participate in one of the three versions of the professional development workshops ("Inspiration", "Inspiration \& Inoculation", or "Inspiration, Inoculation, \& Introduction" groups). Of the total sample of workshop participants, 29 were not included in the analytic sample due to failure to complete either the pre- or the post-workshop surveys. Preliminary analysis indicates no differences in missing data on the pre- or post-workshop surveys across the three groups $\left(\chi_{(\mathrm{df}=2)}^{2}=1.14, p=0.57\right)$.

The number of undergraduate participants $(N=158)$ was nearly equally split across groups $\left(n_{\text {Inspiration }}=53, n_{\text {Inspriation } \& \text { Inoculation }}=57, n_{\text {Inspiration, Inoculation, } \& \text { Introduction }}=48\right)$. Workshop participants were primarily in the first-, second-, or third-year of college, and most were either majoring in Earth Systems science or to a lesser extent Engineering or Biological Science (Supplementary Data 1). Self-reported demographic information revealed that most participants were of European/ Caucasian descent or to a lesser extent of African American descent or of multiple races/ethnicities (Supplementary Data 1). Consistent with the randomized design, preliminary analysis indicated no group differences on any of the pre-workshop academic or demographic variables (Supplementary Data 1 notes).

Procedure. Recruitment and invitation to participate: Undergraduates enrolled at 10 universities were recruited to participate in regional 1-day professional development workshops for women interested in Earth Systems or Environmental Science careers. Advertisements for the professional development workshop were distributed via email. Specifically, emails were distributed by (a) targeted advertisements sent directly to female undergraduates in geoscience-related majors as identified by a university registrar's office, (b) general advertisements sent to geoscience-related distribution lists forwarded by local campus partners (e.g., university faculty or staff), or (c) general advertisements distributed by local campus partners to students in geoscience-related classes. Emailed advertisements emphasized that the 1-day professional development workshop was an opportunity for college women to learn more about Earth Systems and Environmental Science careers, meet peers with similar interest, and network with geoscience professionals. Students interested in participating in the workshop completed a brief online application that asked questions about their gender identity (e.g., woman, transgender woman), university affiliation, college rank (e.g., first-year student), major, interest in pursuing an Earth Systems and Environmental Science career (i.e., yes, no), racial/ethnic identity, and age (i.e., $\leq 17$ years, $\geq 18$ years), as well as a university IRB-approved informed consent form. All applicants who identified as female or a gender minority (transgender woman or gender non-binary/fluid), were $\geq 18$ years of age, who were currently or intended to major in a geosciencerelated field, and who intended to pursue a geoscience-related career received an email invitation to participate in one of the three 1-day professional development workshops held at local university. A blocked randomization procedure was used to assign applicants within each university to a workshop to ensure that applicants from the same university distributed equally across conditions.

Applicants completed online surveys 1 week prior to attending the workshop (i.e., pretest survey), as well as an online survey 3 months after the workshop (i.e., posttest survey). The pretest survey included measures of demographic characteristics, academic interests and performance, mentoring experiences, research experiences, and psychological processes such as science identity, as well as a "getting to know you" survey used for "birds of a feather" matching (see "Measures" section below). The posttest survey was identical to the pretest survey, with the exception that demographic and "getting to know you" questions were omitted. Participants received a small incentive for completing surveys (a \$20 Starbucks E-Gift Card or a \$20 Amazon E-Gift Card for completing each survey). A priori estimates of the sample required to achieve acceptable levels of statistical power (i.e., $\beta=0.80$ ) to detect potential experimental effects on mentoring and mediated effects on persistence intentions were derived using the $\mathrm{G}^{*}$ Power software $\mathrm{v} 3.01^{65}$ and MedPower ${ }^{66}$. The power analyses revealed that a minimum sample size of 130 (or $n=43$ per group) would be required to achieve adequate power to detect the expected experimental and mediated effects. All study procedures were approved by a local IRB (IRB protocol 14-4829H).

Workshops and post-workshop experiences: The study team provided transportation to and from the professional development workshops for all participants. Workshops were developed to test for the critical elements of a successful mentoring and role modeling program (i.e., PROGRESS) aimed at increasing women's persistence in the geosciences $24,29,30$. Workshop content and post-workshop experiences were systematically varied to identify essential elements of mentoring programs, such as distinguishing the unique benefits of role modeling (lower resource intensity) from those of mentee resilience training and follow-up mentoring (higher resource intensity)

Workshop 1 (Inspiration) focused on exposure to geoscience careers via female career role models. The workshop included (1) a brief introduction to Earth Systems and Environmental Sciences careers, as well as a discussion of challenges to success in scientific careers (e.g., implicit bias), (2) a panel session focused on successful professional and personal "Pathways to Earth and Environmental Sciences" featuring a diverse group of female geoscientists, and (3) a panel session focused on "A Day in the Life of an Earth and Environmental Scientist" featuring a diverse group female geoscientists. In addition, Workshop 1 included social activities and ice-breakers leading to an introduction to a website (geosciencewomen.org) and private Facebook page where participants could view inspirational stories of women in the geosciences posted by the project team and continue contact with their peers, respectively. The discussion of implicit bias used here includes interactive exercises on automatic thinking. Stereotyping is defined as automatic thinking applied to social groups. For example, the students participate in a "mock" version of the Implicit Association Task ${ }^{67}$, meant to demonstrate that implicit biases regarding gender and occupations are deeply embedded ${ }^{68}$. The module also explains the concept of stereotype threat and provides specific examples of its impact on academic performance ${ }^{69,70}$. The facilitator emphasizes that recognizing and confronting implicit biases is important, and the module ends with a discussion of how individuals can confront these issues on behalf of themselves or others.

Workshops 2 and 3 ("Inspiration \& Inoculation" and "Inspiration, Inoculation, \& Introductions", respectively) contained identical content on exposure to geoscience careers via female career role models and further included training on how to grow a network of mentoring relationships and overcome obstacles to success in geosciences careers. The workshops included (1) a brief introduction to Earth Systems and Environmental Sciences, as well as a discussion of challenges to success in scientific careers, (2) a panel session focused on successful professional and personal "Pathways to Earth and Environmental Sciences" featuring a diverse group of female geoscientists, (3) a panel session on developing, maintaining, and setting appropriate expectations for successful mentoring relationships in the geosciences featuring a diverse group of female geoscientists, (4) a mentor network mapping and strategic growth activity, and (5) two activities focused on building a toolkit for resilience and success in the geosciences (i.e., emphasizing grit, growth mindset, goal setting for developing mentoring relationships, and practice activities initiating mentoring relationships and developing professional correspondence with professors or other mentors). In addition, Workshops 2 and 3 held a session that introduced a website (geosciencewomen.org) and private Facebook page where participants could view project team posted inspirational stories of women in the geosciences and continue contact with their peers, respectively.

Finally, Workshop 3 participants received a follow-up email within 1 week of completing the workshop introducing them to a local similar female geoscientist. The introductory email set the expectation that the pair should meet on one occasion for up to $1 \mathrm{~h}$. In addition to facilitating the introduction, the email listed three ways that the pair were similar according to their responses to the "getting to know you" survey. All participants and volunteer mentors completed a brief "getting to know you" survey prior to the date of the workshops ${ }^{42}$. A "birds of a feather" procedure was used to both match the pairs and heighten their perceived similarity-a key attribute of initiating successful mentoring relationships ${ }^{57,71,72}$. In addition to matching volunteer mentors with participants based on similarity on three responses from their "getting to know you" survey, proximity (i.e., close enough in proximity to meet in person on one occasion) and disciplinary focus (e.g., Earth science majors with Earth scientists) were also considered but not highlighted in the introductory email. The volunteer mentors were directed to send a follow-up email within 1 week of the introduction to schedule the meeting time and location. Volunteer mentors were offered reimbursement up to $\$ 10$ for inviting their matched potential mentee(s) to meet with them in person for coffee or ice cream.

Measures. Unless otherwise specified, scales were administered at both pretest and posttest.

Outcome: Scientific career persistence intentions. This three-item scale measured the degree to which participant's intended to pursue a scientific career (e.g., "What is the likelihood of you obtaining a science-related degree?", "To what extent do you plan to pursue a science-related graduate degree?", and "To what extent do you plan to pursue a science-related research career?") ${ }^{73}$. Participants rated their agreement with each statement on a Likert scale from 1 (strongly disagree) to 7 (strongly agree). Scientific career persistence intentions scale scores were calculated as the average of responses to the three items, with a higher score indicating a higher level of persistence intentions. Descriptive statistics and reliability estimates are shown in Supplementary Data 2 and 4.

Motivation and coping skills (Mediators): Scientific identity. This 6-item scale (adapted from the original 14-item scale) measured the degree to which participant's think of themselves as a scientist (e.g., "I have come to think of myself as a scientist") ${ }^{74}$. Participants rated their agreement with each statement on a Likert scale from 1 (strongly disagree) to 7 (strongly agree). Scientific identity scale scores were calculated as the average of responses to the six items, with a higher score indicating a higher level of science identity. Descriptive statistics and reliability estimates are shown in Supplementary Data 2 and 4 .

Coping skills. Three two-item subscales measured coping with problems via active coping ("I've been taking action to try to make the situation better"), emotional support seeking coping (e.g., "I've been getting emotional support from others"), and information support seeking coping (e.g., "I've been getting help and advice from other people"). The 3 subscales (6 items in total) were taken from the original 28-item Brief Coping with Problems Experienced scale ${ }^{75,76}$. Participants rated their agreement with each statement on a scale from 1 (I haven't been doing this at all) to 4 (I've been doing this a lot). Active, emotion support seeking, and 
information support seeking coping scale scores were calculated as the average of responses to each two-item subscale, with a higher score indicating a higher level of coping skills. Descriptive statistics and reliability estimates are shown in Supplementary Data 2 and 4.

Mentoring and role modeling: Female STEM career role models. Participants were instructed to think of a career role model as "a person who inspires you, someone with whom you identify emotionally, and someone you wish to emulate. A career role model may or may not be aware of your admiration and may not be aware that he or she is a role model for you"29,40. Participants were asked if they had one or more persons who they considered to be a STEM career role model, and if "Yes," they were asked to list the name, gender, and the occupation of up to three STEM career role model(s). A summary index of the number of female STEM career role models was tabulated based on participant responses, with a range from 0 (no female STEM career role models identified) to 3 (three female STEM career role models identified). Consistent with prior research, the posttest role modeling variable was recoded into two variables representing having one female STEM career role model (one role model $=1$, otherwise $=0$ ) or having multiple female STEM career role models (multiple role models $=1$, otherwise $=0)^{29}$. Descriptive statistics are shown in Supplementary Data 2.

Mentor network size. Participants were instructed to think of a mentor as "someone who provides guidance, assistance, and encouragement on professional and academic issues. A mentor is more than an academic advisor and is someone you turn to for guidance and assistance beyond selecting classes or meeting academic requirements" 30 . With that definition in mind, participants were asked if there were any persons they considered to be a career mentor, and if "Yes," they were asked to list the name and career stage of up to three career mentors. A summary index of the number of career mentors was tabulated based on participant responses, with a range from 0 (no career mentors) to 3 (3 career mentors). Consistent with prior research, the posttest mentor network variable was recoded into two variables representing having one career mentor (one mentor $=1$, otherwise $=0$ ) or having multiple career mentors (multiple mentors $=1$, otherwise $=0)^{30,32,77}$. Descriptive statistics are shown in Supplementary Data 2 .

Quality of mentorship support. Participants who indicated that they had one or more mentors were asked follow-up mentorship support questions for each of their career mentors. This adapted four-item scale measured the degree to which each mentor in their network provided psychosocial support, instrumental support, role modeling support, and overall relationship satisfaction (e.g., "To what extent has your mentor conveyed empathy for your concerns or feelings you have discussed with him or her?") ${ }^{71,78,79}$. Participants rated the degree of support received from each mentor on a scale from 1 (not at all) to 7 (to a large extent). Quality of mentorship support scale scores were derived as the average response across all mentoring items for all mentors, with higher scores indicating higher levels of mentoring support. Descriptive statistics are shown in Supplementary Data 2.

Plan of analysis. Our statistical approach was to fit a path model to our data to test the hypothesized experimental process model shown in Fig. 1. As shown in Fig. 1, we chose to analyze the rank-ordered categorical total number of female role models and total number of mentors variables (i.e., measured from 0 to 3 ) as binary indicators of having one or multiple role models or mentors in order to facilitate the estimation of both direct and indirect (i.e., mediate) effects of the intervention on the outcomes. An alternative, but equivalent, approach would have been to analyze the rank-ordered role modeling and mentoring variables using ordinal logistic regression; however, that approach would have precluded the estimation of indirect effects, which were of central interest to the present study.

Path analysis was conducted in a structural equation modeling framework using maximum likelihood estimation in Mplus version $8.00^{80}$. Consistent with best practices, the adequacy of data-model fit was assessed using a variety of global fit indices, including the $\chi^{2}$ test, RMSEA, CFI, and SRMR ${ }^{81,82}$. Observed fit indices were compared to values representing "good" model fit, such as a non-significant $\chi^{2}$ test, CFI between 0.95 and 1.0, RMSEA between 0.00 and 0.05 (or a 90\% CI that included 0.05 but did not include 0.10 ), or SRMR between 0.00 and 0.08 . In addition, given our research interest in mediation and the non-normal distribution of some variables used in the analysis, we implemented a robust bootstrapping approach with 5000 iterations to estimate bias-corrected CIs around all modelbased direct and indirect effects ${ }^{83-85}$. The bootstrapping approach does not require the assumption of normality to accurately estimate CIs around parameter estimates for the model (i.e., it is robust to non-normality due to being a distribution-free estimation procedure).

\section{Data availability}

The datasets generated during and analyzed during the current study are available in the Mountain Scholars of Colorado and Wyoming repository (https://doi.org/10.25675/ $10217 / 201607)^{86}$.

Received: 11 March 2020; Accepted: 18 June 2020; Published online: 13 August 2020

\section{References}

1. Bernard, R. E. \& Cooperdock, E. H. G. No progress on diversity in 40 years. Nat. Geosci. 11, 292-295 (2018).

2. Freeman, J. LGBTQ scientists are still left out. Nature 559, 27-28 (2018).

3. Haacker, R. From recruitment to retention. Nat. Geosci. 8, 577-578 (2015).

4. Shaw, A. K. \& Stanton, D. E. Leaks in the pipeline: separating demographic inertia from ongoing gender differences in academia. Proc. R. Soc. B Biol. Sci. 279, 3736-3741 (2012).

5. Larivière, V., Ni, C., Gingras, Y., Cronin, B. \& Sugimoto, C. R. Bibliometrics: global gender disparities in science. Nat. News 504, 211 (2013).

6. Hughes, B. E. Coming out in STEM: factors affecting retention of sexual minority STEM students. Sci. Adv. 4, eaao6373 (2018).

7. Bennett, R. A. \& Lamb, D. A. Accessibility and innovation. Nat. Geosci. 9, 263 (2016)

8. Gilley, B., Atchison, C., Feig, A. \& Stokes, A. Impact of inclusive field trips. Nat. Geosci. 8, 579 (2015).

9. Ford, H. L., Brick, C., Azmitia, M., Blaufuss, K. \& Dekens, P. Women from some under-represented minorities are given too few talks at world's largest Earth-science conference. Nature 576, 32-35 (2019).

10. Ford, H. L., Brick, C., Blaufuss, K. \& Dekens, P. S. Gender inequity in speaking opportunities at the American Geophysical Union Fall Meeting. Nat. Commun. 9, 1358 (2018).

11. Popp, A. L., Lutz, S. R., Khatamis, S., van Emmerik, T. H. M. \& Knoben, W. J. M. A Global survey on the perceptions and impacts of gender inequality in the Earth and space sciences. Earth Space Sci. 6, 1460-1468 (2019).

12. Atchison, C. L. \& Libarkin, J. C. Professionally held perceptions about the accessibility of the geosciences. Geosphere. 12, 1154-1165 (2016).

13. Dutt, K., Pfaff, D. L., Bernstein, A. F., Dillard, J. S. \& Block, C. J. Gender differences in recommendation letters for postdoctoral fellowships in geoscience. Nat. Geosci. 9, 805 (2016).

14. Woolston, C. How four winning mentors help to build skills and dispel doubt. Nature 565, 255-257 (2019).

15. Al-Gazali, L. et al. Scientists of the world speak up for equality. Nature 495, 35-38 (2013).

16. Glessmer, M., Adams, A., Hastings, M. \& Barnes, R. in The Mentoring Continuum: From Graduate School Through Tenure (ed. Wright, G.) (Syracuse University Graduate School Press, Syracuse, NY, 2015).

17. National Academies of Sciences Engineering \& Medicine. The Science of Effective Mentorship in STEMM (eds Byars-Winston, A. \& Dahlberg, M. L.) (The National Academies Press, Washington, DC, 2019).

18. National Academies of Sciences Engineering and Medicine. Undergraduate Research Experiences for STEM Students: Successes, Challenges, and Opportunities (eds Gentile, J., Brenner, K. \& Stephens, A.) (The National Academies Press, Washington, DC, 2017).

19. Newton, A. Plugging the leaks. Nat. Geosci. 5, 522 (2012)

20. Pfund, C., Pribbenow, C. M., Branchaw, J., Lauffer, S. M. \& Handelsman, J. The merits of training mentors. Science 311, 473-474 (2006).

21. Linn, M. C., Palmer, E., Baranger, A., Gerard, E. \& Stone, E. Undergraduate research experiences: Impacts and opportunities. Science 347, 6222 (2015).

22. Fridkis-Hareli, M. A mentoring program for women scientists meets a pressing need. Nat. Biotechnol. 29, 287-288 (2011).

23. Kuhn, C. \& Castaño, Z. Boosting the career development of postdocs with a peer-to-peer mentor circles program. Nat. Biotechnol. 34, 781-783 (2016).

24. Fischer, E. V. et al. Welcoming women into the geosciences. Eos https://doi. org/10.1029/2018EO095017 (2018).

25. Maton, K. I. \& Hrabowski, F. A. Increasing the number of African American PhDs in the sciences and engineering: a strengths-based approach. Am. Psychol. 59, 547-556 (2004).

26. Wilson, Z. S. et al. Hierarchical mentoring: a transformative strategy for improving diversity and retention in undergraduate STEM disciplines. J. Sci. Educ. Technol. 21, 148-156 (2011).

27. Adams, A. S., Steiner, A. L. \& Wiedinmyer, C. The earth science women's network (ESWN): community-driven mentoring for women in the atmospheric sciences. Bull. Am. Meteorol. Soc. 97, 345-354 (2016).

28. Avallone, L. M., Hallar, A. G., Thiry, H. \& Edwards, L. M. Supporting the retention and advancement of women in the Atmospheric Sciences: what women are saying. Bull. Am. Meteorol. Soc. 94, 1313-1316 (2013).

29. Hernandez, P. R. et al. Role modeling is a viable retention strategy for undergraduate women in the geosciences. Geosphere 14, 2585-2593 (2018).

30. Hernandez, P. R. et al. Promoting professional identity, motivation, and persistence: benefits of an informal mentoring program for female undergraduate students. PLOS ONE 12, e0187531 (2017).

31. Packard, B. W. L., Walsh, L. \& Seidenberg, S. Will that be one mentor or two? A cross-sectional study of women's mentoring during college. Mentoring Tutoring Partnership Learn. 12, 71-85 (2004).

32. Higgins, M. C. The more, the merrier? Multiple developmental relationships and work satisfaction. J. Manage. Dev. 19, 277-296 (2000). 
33. Aikens, M. L. et al. Race and gender differences in undergraduate research mentoring structures and research outcomes. CBE Life Sci. Educ. 16, 2 (2017)

34. Graham, M. J., Frederick, J., Byars-Winston, A., Hunter, A.-B. \& Handelsman, J. Increasing persistence of college students in STEM. Science 341, 1455-1456 (2013).

35. Dennehy, T. C. \& Dasgupta, N. Female peer mentors early in college increase women's positive academic experiences and retention in engineering. Proc. Natl Acad. Sci. 114, 5964-5969 (2017).

36. Dasgupta, $\mathrm{N}$. Ingroup experts and peers as social vaccines who inoculate the self-concept: the stereotype inoculation model. Psychol. Inquiry 22, 231-246 (2011).

37. Drury, B. J., Siy, J. O. \& Cheryan, S. When do female role models benefit women? The importance of differentiating recruitment from retention in STEM. Psychol. Inquiry 22, 265-269 (2011).

38. Dawson, A. E., Bernstein, B. L. \& Bekki, J. M. Providing the psychosocial benefits of mentoring to women in STEM: careerWISE as an online solution. New Directions Higher Educ. 171, 53-62 (2015).

39. Good, C., Rattan, A. \& Dweck, C. S. Why do women opt out? Sense of belonging and women's representation in mathematics. J. Pers. Soc. Psychol. 102, 700-717 (2012)

40. Lockwood, P. "Someone like me can be successful": do college students need same-sex gender role models? Psychol. Women Q. 30, 36-46 (2006).

41. Higgins, M. C. \& Kram, K. E. Reconceptualizing mentoring at work: a developmental network perspective. Acad. Manage. Rev. 26, 264-288 (2001).

42. Gehlbach, H. et al. Creating birds of similar feathers: leveraging similarity to improve teacher-student relationships and academic achievement. J. Educ. Psychol. 108, 342-352 (2016).

43. Lockwood, P. \& Kunda, Z. Superstars and me: predicting the impact of role models on the self. J. Pers. Soc. Psychol. 73, 91-103 (1997).

44. Cheryan, S., Siy, J. O., Vichayapai, M., Drury, B. J. \& Kim, S. Do female and male role models who embody STEM stereotypes hinder women's anticipated success in STEM? Soc. Psychol. Pers. Sci. 2, 656-664 (2011).

45. Herrmann, S. D. et al. The effects of a female role model on academic performance and persistence of women in STEM courses. Basic Appl. Soc. Psychol. 38, 258-268 (2016).

46. Stout, J. G., Dasgupta, N., Hunsinger, M. \& McManus, M. A. STEMing the tide: using ingroup experts to inoculate women's self-concept in science, technology, engineering, and mathematics (STEM). J. Pers. Soc. Psychol. 100, 255-270 (2011).

47. Johnson, W. B., Rose, G. L. \& Schlosser, L. Z. in The Blackwell Handbook of Mentoring: A Multiple Perspectives Approach (eds Allen, T. D. \& Eby, L. T.) 49-70 (Blackwell, Oxford, 2007).

48. Jacobi, M. Mentoring and undergraduate academic success: a literature review. Rev. Educ. Res. 61, 505-532 (1991)

49. Crisp, G. \& Cruz, I. Mentoring college students: a critical review of the literature between 1990 and 2007. Res. Higher Educ. 50, 525-545 (2009).

50. Gershenfeld, S. A review of undergraduate mentoring programs. Rev. Educ. Res. 84, 365-391 (2014).

51. Komro, K. A., Flay, B. R., Biglan, A. \& Wagenaar, A. C. Research design issues for evaluating complex multicomponent interventions in neighborhoods and communities. Tran.sl Behav. Med. 6, 153-159 (2016).

52. Shadish, W., Cook, T. \& Campbell, D. Experimental and Quasi-experimental Designs for Generalized Causal Inference (Houghton Mifflin Company, Boston, 2002).

53. Dweck, C. S., Walton, G. M. \& Cohen, G. L. Academic Tenacity: Mindsets and Skills that Promote Long-term Learning (Bill \& Melinda Gates Foundation, 2014).

54. Good, C., Aronson, J. \& Inzlicht, M. Improving adolescents' standardized test performance: an intervention to reduce the effects of stereotype threat. J. Appl. Dev. Psychol. 24, 645-662 (2003).

55. Moss-Racusin, C. A., Dovidio, J. F., Brescoll, V. L., Graham, M. J. \& Handelsman, J. Science faculty's subtle gender biases favor male students. Proc. Natl Acad. Sci. 109, 16474-16479 (2012).

56. Blake-Beard, S., Bayne, M. L., Crosby, F. J. \& Muller, C. B. Matching by race and gender in mentoring relationships: keeping our eyes on the prize. J. Soc. Issues 67, 622-643 (2011).

57. Hernandez, P. R., Estrada, M., Woodcock, A. \& Schultz, P. W. Protégé perceptions of high mentorship quality depend on shared values more than on demographic match. J. Exp. Educ. 85, 450-468 (2016).

58. Conger, A. J. A revised definition for suppressor variables: a guide to their identification and interpretation. Educ. Psychol. Measurement 34, 35-46 (1974).

59. Morganson, V. J., Jones, M. P. \& Major, D. A. Understanding women's underrepresentation in science, technology, engineering, and mathematics: the role of social coping. Career Dev. Q. 59, 169-179 (2010).

60. Rosenthal, L., London, B., Levy, S. R. \& Lobel, M. The roles of perceived identity compatibility and social support for women in a single-sex STEM program at a co-educational university. Sex Roles 65, 725-736 (2011).
61. Cartier, K. M. S. Body-based jargon can be harassment when it turns sexual. Eos https://doi.org/10.1029/2020EO138285 (2020).

62. Dutt, K. Race and racism in the geosciences. Nat. Geosci. 13, 2-3 (2020).

63. Mattheis, A., Murphy, M. \& Marin-Spiotta, E. Examining intersectionality and inclusivity in geosciences education research: a synthesis of the literature 2008-2018. J. Geosci. Educ. 67, 505-517 (2019).

64. Marín-Spiotta, E., Schneider, B. \& Holmes, M. A. Steps to building a notolerance culture for sexual harassment. Eos https://doi.org/10.1029/ 2016EO044859 (2016)

65. Faul, F., Erdfelder, E., Lang, A.-G. \& Buchner, A. G*Power3: a flexible statistical power analysis program for the social, behavioral, and biomedical sciences. Behav. Res. Methods. 39, 175-191 (2007).

66. Kenny, D. A. MedPower: an interactive tool for the estimation of power in tests of mediation. http://davidakenny.net/cm/mediate.htm (2017)

67. Greenwald, A. G., McGhee, D. E. \& Schwartz, J. L. K. Measuring individual differences in implicit cognition: the implicit association test. J. Pers. Soc. Psychol. 74, 1464-1480 (1998).

68. Rudman, L. A. \& Glick, P. Prescriptive gender stereotypes and backlash toward agentic women. J Soc Issues 57, 743-62. (2001).

69. Steele, C. M. \& Aronson, J. Stereotype threat and the intellectual test performance of African Americans. J. Pers. Soc. Psychol. 69, 797-811 (1995).

70. Spencer, S. J., Steele, C. M. \& Quinn, D. M. Stereotype threat and women's math performance. J. Exp. Soc. Psychol. 35, 4-28 (1999).

71. Ensher, E. A. \& Murphy, S. E. Effects of race, gender, perceived similarity, and contact on mentor relationships. J. Vocational Behav. 50, 460-481 (1997).

72. de Janasz, S. C. \& Godshalk, V. M. The role of e-mentoring in proteges' learning and satisfaction. Group Organ. Manage. 38, 743-774 (2013).

73. Woodcock, A., Hernandez, P. R. \& Schultz, P. W. Diversifying science: intervention programs moderate the effect of stereotype threat on motivation and career choice. Soc. Psychol. Pers. Sci. 7, 184-192 (2015).

74. Chemers, M. M., Zurbriggen, E. L., Syed, M., Goza, B. K. \& Bearman, S. The role of efficacy and identity in science career commitment among underrepresented minority students. J. Soc. Issues 67, 469-491 (2011).

75. Carver, C. S. You want to measure coping but your protocol's too long: consider the brief COPE. Int. J. Behav. Med. 4, 92 (1997).

76. Carver, C. S., Scheier, M. F. \& Weintraub, J. K. Assessing coping strategies: a theoretically based approach. J. Pers. Soc. Psychol. 56, 267-283 (1989).

77. Downing, R. A., Crosby, F. J. \& Blake-Beard, S. The perceived importance of developmental relationships on women undergraduates' pursuit of science. Psychol. Women Q. 29, 419-426 (2005).

78. Tenenbaum, H. R., Crosby, F. J. \& Gliner, M. D. Mentoring relationships in graduate school. J. Vocational Behav. 59, 326-341 (2001).

79. Hoyt, C. L., Burnette, J. L. \& Innella, A. N. I can do that: the impact of implicit theories on leadership role model effectiveness. Pers. Soc. Psychol. Bull. 38, 257-268 (2012).

80. Muthén, B. O. \& Muthén, L. K. Mplus User's Guide 8.0 edn (Muthén \& Muthén, Los Angeles, CA, 1998-2017).

81. Hu, L. \& Bentler, P. M. Cutoff criteria for fit indices in covariance structure analysis: conventional criteria versus new alternatives. Struct. Equation Model. 6, 1-55 (1999).

82. Kline, R. B. Principles and Practice of Structural Equation Modeling 4 edn (ed. Kenny, D.) (Guilford Press, New York, NY, 2016)

83. Stine, R. An introduction to bootstrap methods: examples and ideas. Sociol. Methods Res. 18, 243-291 (1989).

84. Effron, B. \& Tibshirani, R. J. An Introduction to the Boostrap (Chapman and Hall, Boca Raton, FL, 1993).

85. MacKinnon, D. P., Lockwood, C. M., Hoffman, J. M., West, S. G. \& Sheets, V. A comparison of methods to test mediation and other intervening variable effects. Psychol. Methods. 7, 83-104 (2002).

86. Dataset associated with "Inspiration, inoculation, and introductions are all critical to successful mentorship for undergraduate women pursuing geoscience careers". https://mountainscholar.org/handle/10217/201607 (2020).

\section{Acknowledgements}

This work was supported by the National Science Foundation (DUE-1431795, DUE1431823, and DUE-1460229).

\section{Author contributions}

P.R.H., A.S.A., R.T.B., S.M.C., and E.V.F. conceptualized the project; P.R.H., W.D., H.H., and E.V.F. wrote the original draft; P.R.H., W.D., and H.H. formally analyzed the data; P.R.H. and I.P. created figures; and all authors edited and reviewed the manuscript.

\section{Competing interests}

The authors declare no competing interests. 


\section{Additional information}

Supplementary information is available for this paper at https://doi.org/10.1038/s43247020-0005-y.

Correspondence and requests for materials should be addressed to P.R.H.

Peer review information Primary handling editor: Heike Langenberg.

Reprints and permission information is available at http://www.nature.com/reprints

Publisher's note Springer Nature remains neutral with regard to jurisdictional claims in published maps and institutional affiliations. (c) (i) Open Access This article is licensed under a Creative Commons Attribution 4.0 International License, which permits use, sharing, adaptation, distribution and reproduction in any medium or format, as long as you give appropriate credit to the original author(s) and the source, provide a link to the Creative Commons license, and indicate if changes were made. The images or other third party material in this article are included in the article's Creative Commons license, unless indicated otherwise in a credit line to the material. If material is not included in the article's Creative Commons license and your intended use is not permitted by statutory regulation or exceeds the permitted use, you will need to obtain permission directly from the copyright holder. To view a copy of this license, visit http://creativecommons.org/licenses/by/4.0/.

(C) The Author(s) 2020 